\title{
ANALYTIC INTEGRABILITY OF HAMILTONIAN SYSTEMS WITH EXCEPTIONAL POTENTIALS
}

\author{
JAUME LLIBRE ${ }^{1}$ AND CLAUDIA VALLS ${ }^{3}$
}

Abstract. We study the existence of analytic first integrals of the complex Hamiltonian systems of the form

$$
H=\frac{1}{2} \sum_{i=1}^{2} p_{i}^{2}+V_{l}\left(q_{1}, q_{2}\right)
$$

with the homogeneous polynomial potential

$V_{l}\left(q_{1}, q_{2}\right)=\alpha\left(q_{2}-i q_{1}\right)^{l}\left(q_{2}+i q_{1}\right)^{k-l}, \quad l=0, \ldots, k, \quad \alpha \in \mathbb{C} \backslash\{0\}$ of degree $k$ called exceptional potentials. In Remark 2.1 of J. Math. Phys. 46 (2005), 062901, the authors state: The exceptional potentials $V_{0}, V_{1}, V_{k-1}, V_{k}$ and $V_{k / 2}$ when $k$ is even are integrable with a second polynomial first integral. However nothing is known about the integrability of the remaining exceptional potentials.

Here we prove that the exceptional potentials with $k$ even different from $V_{0}, V_{1}, V_{k-1}, V_{k}$ and $V_{k / 2}$, have no independent analytic first integral different from the Hamiltonian one.

Additionally in the cases $V_{2}$ and $V_{k-2}$ with $k$ either even or odd we show that they do not have rational first integrals independent of the Hamiltonian.

\section{INTRODUCTION AND STATEMENT OF THE MAIN RESULTS}

Ordinary differential equations in general and Hamiltonian systems in particular play a very important role in many branches of the applied sciences. The question whether a differential system admits a first integral is of fundamental importance as first integrals give conservation laws for the model and that enables to lower the dimension of the system. Moreover knowing a sufficient number of first integrals allows to solve the system explicitly. Until the end of the 19th century the majority of scientists thought that the equations of classical mechanics were integrable and finding the first integrals was mainly a problem of computation. In fact, now we know that the integrability is a nongeneric phenomenon inside the class of Hamiltonian systems (see [3]), and in general it is very hard to determine whether a given Hamiltonian system is integrable or not.

2010 Mathematics Subject Classification. 37J35.

Key words and phrases. Hamiltonian system with 2-degrees of freedom, homogeneous potentials of degree $\mathrm{k}$, exceptional potentials, integrability. 
In this work we are concerned with the integrability of the natural Hamiltonian systems defined by a Hamiltonian function of the form

$$
H=\frac{1}{2} \sum_{i=1}^{2} p_{i}^{2}+V\left(q_{1}, q_{2}\right),
$$

where $V\left(q_{1}, q_{2}\right) \in \mathbb{C}\left[q_{1}, q_{2}\right]$ is a homogeneous polynomial potential of degree $k$. As usual $\mathbb{C}\left[q_{1}, q_{2}\right]$ is the ring of polynomial functions over $\mathbb{C}$ in the variables $q_{1}$ and $q_{2}$. To be more precise we consider the following system of four differential equations

$$
\dot{q}_{i}=p_{i}, \quad \dot{p}_{i}=-\frac{\partial V}{\partial q_{i}}, \quad i=1,2 .
$$

Let $A=A(\mathbf{q}, \mathbf{p})$ and $B=B(\mathbf{q}, \mathbf{p})$ be two functions, where $\mathbf{p}=\left(p_{1}, p_{2}\right)$ and $\mathbf{q}=\left(q_{1}, q_{2}\right)$. We define the Poisson bracket of $A$ and $B$ as

$$
\{A, B\}=\sum_{i=1}^{2}\left(\frac{\partial A}{\partial q_{i}} \frac{\partial B}{\partial p_{i}}-\frac{\partial A}{\partial p_{i}} \frac{\partial B}{\partial q_{i}}\right) .
$$

The functions $A$ and $B$ are in involution if $\{A, B\}=0$. A non-constant function $F=F(\mathbf{q}, \mathbf{p})$ is a first integral for the Hamiltonian system (2) if it is in involution with the Hamiltonian function $H$, i.e. $\{H, F\}=0$. Since the Poisson bracket is antisymmetric it follows that $H$ itself is always a first integral. A 2-degree of freedom Hamiltonian system (2) is completely or Liouville integrable if it has 2 functionally independent first integrals $H$ and $F$. As usual $H$ and $F$ are functionally independent if their gradients are linearly independent at all points of $\mathbb{C}^{4}$ except perhaps in a zero Lebesgue measure set.

Let $\mathrm{PO}_{2}(\mathbb{C})$ denote the group of $2 \times 2$ complex matrices $A$ such that $A A^{T}=\alpha \mathrm{Id}$, where Id is the $2 \times 2$ identity matrix and $\alpha \in \mathbb{C} \backslash\{0\}$. The potentials $V_{1}(\mathbf{q})$ and $V_{2}(\mathbf{q})$ are equivalent if there exists a matrix $A \in$ $\mathrm{PO}_{2}(\mathbb{C})$ such that $V_{1}(\mathbf{q})=V_{2}(A \mathbf{q})$. Therefore we divide all potentials into equivalent classes. In what follows a potential means a class of equivalent potentials in the above sense. This definition of equivalent potentials is motivated by the following simple observation (for a proof see [1]). Let $V_{1}$ and $V_{2}$ be two equivalent potentials. If the Hamiltonian system (2) with the potential $V_{1}$ is integrable, then it is also integrable with the potential $V_{2}$.

It was shown in [2] that among all equivalent potentials one can always choose a representative $V$ such that the polynomial $V$ has one root in an arbitrary point of $\mathbb{C P}^{1} \backslash\{[1:+i],[1:-i]\}$. This is always possible except for cases when all linear factors of $V$ have the form $q_{2} \pm i q_{1}$, that is, if the potential $V$ is of the form

$$
V=V_{l}=\alpha\left(q_{2}-i q_{1}\right)^{l}\left(q_{2}+i q_{1}\right)^{k-l}, \quad l=0, \ldots, k, \quad \alpha \in \mathbb{C} \backslash\{0\} .
$$

These potentials are called exceptional. It was proved in [1] that the exceptional potentials $V_{0}, V_{1}, V_{k-1}, V_{k}$ and $V_{k / 2}$ when $k$ is even are 
integrable. It is easy to find that for these exceptional potentials the additional polynomial first integral is:

$$
\begin{aligned}
I_{0} & =p_{1}-i p_{2}, \quad I_{1}=k\left(p_{1}-i p_{2}\right)^{2}-4 \alpha\left(q_{2}+i q_{1}\right)^{k}, \\
I_{k-1} & =k\left(p_{1}+i p_{2}\right)^{2}-4 \alpha\left(q_{2}-i q_{1}\right)^{k}, \quad I_{k}=p_{1}+i p_{2},
\end{aligned}
$$

and when $k$ is even

$$
I_{k / 2}=q_{2} p_{1}-q_{1} p_{2} \text {. }
$$

It is also claimed in [2] and [1] that nothing is known about the integrability of the remaining exceptional potentials. In this paper we focus on these remaining exceptional potentials. We restrict to the potentials $V_{l}$ with $l=2, \ldots, k / 2-1, k / 2+1, \ldots, k-2$ and $k$ even. Note that if $k \leq 4$ all the exceptional potentials are integrable with polynomial first integrals. So we will focus on the case $k \geq 5$.

System (2) becomes

$$
\begin{aligned}
& \dot{q}_{1}=p_{1}, \\
& \dot{q}_{2}=p_{2} \\
& \dot{p}_{1}=\alpha i\left[(2 l-k) q_{2}+i k_{1} q_{1}\right]\left(q_{2}-i q_{1}\right)^{l-1}\left(q_{2}+i q_{1}\right)^{k-l-1}, \\
& \dot{p}_{2}=-\alpha i\left[(2 l-k) q_{1}-i k q_{2}\right]\left(q_{2}-i q_{1}\right)^{l-1}\left(q_{2}+i q_{1}\right)^{k-l-1} .
\end{aligned}
$$

Our main results are the following.

Theorem 1. System (3) with $k \geq 6$ even and $l=2, \ldots, k / 2-1, k / 2+$ $1, \ldots, k-2$ does not admit an additional analytic first integral independent of the Hamiltonian one.

The proof of Theorem 1 is given in section 3. We state the following conjecture.

Conjecture 1. System (3) with $k \geq 5$ odd and $l=2, \ldots, k-2$ does not admit an additional analytic first integral independent of the Hamiltonian one.

In the case in which $l=2$ or $l=k-2$ with $k$ being either even or odd, we can also prove with different techniques the non-existence of rational first integrals.

Theorem 2. System (3) with $l=2$ or $l=k-2$ and $k \geq 5$ does not admit an additional rational first integral independent of the Hamiltonian one.

The proof of Theorem 2 is given in section 2 .

\section{Weight-homogeneous Polynomial diffEREntial System AND PROOF OF THEOREM 2}

We consider polynomial differential system of the form

$$
\frac{d \mathbf{x}}{d t}=\dot{\mathbf{x}}=\mathbf{P}(\mathbf{x}), \quad \mathbf{x}=\left(x_{1}, x_{2}, x_{3}, x_{4}\right) \in \mathbb{C}^{4}
$$


with $\mathbf{P}(\mathbf{x})=\left(P_{1}(\mathbf{x}), P_{2}(\mathbf{x}), P_{3}(\mathbf{x}), P_{4}(\mathbf{x})\right.$ and $P_{i} \in \mathbb{C}\left[x_{1}, x_{2}, x_{3}, x_{4}\right]$ for $i=1,2,3,4$. As usual $\mathbb{N}, \mathbb{R}$ and $\mathbb{C}$ denote the sets of positive integers, real and complex numbers, respectively; and $\mathbb{C}\left[x_{1}, x_{2}, x_{3}, x_{4}\right]$ denotes the polynomial ring over $\mathbb{C}$ in the variables $x_{1}, x_{2}, x_{3}, x_{4}$. Here $t$ can be real or complex.

We say that system (4) is weight-homogeneous if there exist $\mathbf{s}=$ $\left(s_{1}, s_{2}, s_{3}, s_{4}\right) \in \mathbb{N}^{4}$ and $d \in \mathbb{N}$ such that for arbitrary $a \in \mathbb{R}^{+}=\{a \in$ $\mathbb{R}, a>0\}$ we have

$$
P_{i}\left(a^{s_{1}} x_{1}, a^{s_{2}} x_{2}, a^{s_{3}} x_{3}, a^{s_{4}} x_{4}\right)=a^{s_{i}-1+d} P_{i}\left(x_{1}, x_{2}, x_{3}, x_{4}\right),
$$

for $i=1,2,3,4$. We call $\mathbf{s}=\left(s_{1}, s_{2}, s_{3}, s_{4}\right)$ as the weight exponent of system (4) and $d$ as weight degree with respect to the weight exponent s.

We say that a polynomial $F\left(x_{1}, x_{2}, x_{3}, x_{4}\right)$ is a weight-homogeneous polynomial with weight exponent $\mathbf{s}=\left(s_{1}, s_{2}, s_{3}, s_{4}\right)$ and weight degree $d$ if

$$
F\left(a^{s_{1}} x_{1}, a^{s_{2}} x_{2}, a^{s_{3}} x_{3}, a^{s_{4}} x_{4}\right)=a^{d} F\left(x_{1}, x_{2}, x_{3}, x_{4}\right),
$$

for all $a>0$.

The following well-known proposition (easy to prove) reduces the study of the existence of analytic first integrals of a weight-homogeneous polynomial differential system (4) to the study of the existence of a weight-homogeneous polynomial first integrals.

Proposition 3. Let $H$ be an analytic function and let $H=\sum_{i} H_{i}$ be its decomposition into weight-homogeneous polynomials of weight degree $i$ with respect to the weight exponent $\mathbf{s}$. Then $H$ is an analytic first integral of the weight-homogeneous polynomial differential system (4) with weight exponent $\mathbf{s}$ if and only if each weight-homogeneous part $H_{i}$ is a first integral of system (4) for all $i$.

We introduce the change of variables

$$
x_{1}=p_{1}+i p_{2}, \quad x_{2}=p_{1}-i p_{2}, \quad y_{1}=q_{1}+i q_{2}, \quad y_{2}=q_{1}-i q_{2} .
$$

In these new variables system (3) becomes

$$
\begin{aligned}
& \dot{x}_{1}=2 \alpha(l-k)(-1)^{l} i^{k} y_{1}^{l} y_{2}^{k-l-1}, \\
& \dot{x}_{2}=-2 \alpha l(-1)^{l} i^{k} y_{1}^{l-1} y_{2}^{k-l}, \\
& \dot{y}_{1}=x_{1}, \\
& \dot{y}_{2}=x_{2} .
\end{aligned}
$$

Note that system (5) is Hamiltonian with Hamiltonian

$$
H=\frac{x_{1} x_{2}}{2}+\alpha(-1)^{l} i^{k} y_{1}^{l} y_{2}^{k-l}, \quad \alpha \in \mathbb{C} \backslash\{0\} .
$$

It is easy to check that system (5) is a weight-homogeneous polynomial differential system with weight exponent $s=\left(s_{1}, s_{2}, s_{3}, s_{4}\right)$ where

(6) $s_{1}=l(k+1)+1, \quad s_{2}=(l-1)(k+1), \quad s_{3}=k+l+1, \quad s_{4}=l-1$ 
and weight degree $d=1+k(l-1)$. Note that since $l \geq 2$ we have that $s_{1}, s_{2}, s_{3}, s_{4}, d \in \mathbb{N}$.

From Proposition 3 and the observation above it follows that for proving the existence of non-existence of analytic first integrals of system (5) it is sufficient to show the existence or non-existence of weighthomogeneous polynomial first integrals with weight exponents given in (6).

We recall that in the case in which $k$ is even, we can be more precise and it is clear that system (5) is a weight-homogeneous polynomial differential system with weight exponent $(1,1, k / 2, k / 2)$ and weight degree $d=k / 2$.

Proof of Theorem 2. Instead of Proving Theorem 2 we will prove the following theorem which is equivalent to Theorem 2 .

Theorem 4. System (5) with $l=2$ or with $l=k-2$ does not admit an additional rational first integral.

We will only prove the case $l=k-2$ because the proof of the case $l=2$ is exactly the same interchanging the roles of $y_{1}$ with $y_{2}$ and of $x_{1}$ with $x_{2}$. The proof follows directly from the following theorem which is Theorem 2.4 in [2].

Theorem 5. The Hamiltonian system (1) with potential $V=q_{2}^{2} \tilde{V}\left(q_{1}, q_{2}\right)$ where $\operatorname{deg} \tilde{V}=k-2$ and $\tilde{V}\left(q_{1}, 0\right) \neq 0$, does not admit an additional rational first integral.

We note that in the case $l=k-2$ we have

$$
V\left(y_{1}, y_{2}\right)=\alpha y_{2}^{2} y_{1}^{k-2}=\alpha y_{2}^{2} \tilde{V}\left(y_{1}, y_{2}\right),
$$

where $\operatorname{deg} \tilde{V}=k-2, \tilde{V}\left(y_{1}, y_{2}\right)=\tilde{V}\left(y_{1}, 0\right)=\alpha y_{1}^{k-2}$. It follows directly from Theorem 5 that the Hamiltonian system with potential in (7) does not admit an additional rational first integral independent of the Hamiltonian one. This concludes the proof.

\section{Proof of Theorem 1}

In this section we will prove the following equivalent result to Theorem 1 .

Theorem 6. System (5) with $k \geq 6$ even and $l=2, \ldots, \frac{k}{2}-1, \frac{k}{2}+$ $1, \ldots k-2$ does not admit an additional analytic first integral.

We first observe that we only need to prove Theorem 6 for the cases $l=2, \ldots, \frac{k}{2}-1$, because the proof of the cases $l=\frac{k}{2}+1, \ldots, k-2$ is exactly the same interchanging the roles of $x_{1}$ with $x_{2}$, and $y_{1}$ with $y_{2}$.

Before going into the technicalities of the proof of Theorem 6, we would like to highlight the main idea behind the proof. First we shall restrict system (5) to the zero level of the first integral $H$, which is 
a polynomial function. The restriction to this level set gives rise to a nontrivial rational first integral $\bar{F}$ of the restricted system. To be more precise, $\bar{F}\left(y_{1}, y_{2}, x_{1}\right)$ is a polynomial in the variables $y_{1}, y_{2}, x_{1}$ and $x_{1}^{-1}$. So, it can be written in the following form:

$$
\bar{F}=\sum_{j=-\ell}^{m} g_{j}\left(y_{1}, y_{2}\right) x_{1}^{j} .
$$

We recall again that system (5) is a weight-homogeneous polynomial differential system with weight exponent $(1,1, k / 2, k / 2)$ and weight degree $d=k / 2$. From section 3 it follows that for proving Theorem 6 it is sufficient to show that this system has no weight-homogeneous polynomial first integrals with weight exponent $(1,1, k / 2, k / 2)$.

Let $F=F\left(y_{1}, y_{2}, x_{1}, x_{2}\right) \in \mathbb{C}\left[y_{1}, y_{2}, x_{1}, x_{2}\right]$ be a weight-homogeneous polynomial first integral of system (5) with weight exponent $(1,1, k / 2, k / 2)$ and weight degree $d=\frac{k}{2} n$ with $n \geq 1$. We can express it as

$$
F=\sum_{l_{1}+l_{2}+\frac{k}{2} l_{3}+\frac{k}{2} l_{4}=\frac{k}{2} n} F_{l_{1}, l_{2}, l_{3}, l_{4}} y_{1}^{l_{1}} y_{2}^{l_{2}} x_{1}^{l_{3}} x_{2}^{l_{4}} .
$$

The function $F$ cannot depend only on $y_{1}$ and $y_{2}$. Indeed, if $F=$ $F\left(y_{1}, y_{2}\right)$ then from (5) we get

$$
x_{1} \frac{\partial F}{\partial y_{1}}+x_{2} \frac{\partial F}{\partial y_{2}}=0,
$$

and consequently $F$ is a constant. So $F$ depends on $x_{1}$ or $x_{2}$, and thus $n \geq 2$.

We study the first integral $F$ on the level set $H=0$ by eliminating, for example $x_{2}$ as follows:

$$
x_{2}=-\frac{2 \alpha(-1)^{l+k / 2} y_{1}^{l} y_{2}^{k-l}}{x_{1}} .
$$

Thus, we end up with the following system:

$$
\begin{aligned}
& \dot{y}_{1}=x_{1}, \\
& \dot{y}_{2}=-\frac{2 \alpha(-1)^{l+k / 2} y_{1}^{l} y_{2}^{k-l}}{x_{1}}, \\
& \dot{x}_{1}=-2 \alpha(-1)^{l+k / 2}(k-l) y_{1}^{l} y_{2}^{k-l-1} .
\end{aligned}
$$

Note that the restriction of the polynomial first integral $F$ to the level set $H=0$ can be written as

$$
\begin{aligned}
\bar{F} & =\sum_{l_{1}+l_{2}+\frac{k}{2} l_{3}+\frac{k}{2} l_{4}=\frac{k}{2} n} \bar{F}_{l_{1}, l_{2}, l_{3}} y_{1}^{l_{1}} y_{2}^{l_{2}} x_{1}^{l_{3}}\left(-\frac{2 \alpha(-1)^{l+k / 2} y_{1}^{l} y_{2}^{k-l}}{x_{1}}\right)^{l_{4}} \\
& =\sum_{j=-n}^{n} \bar{F}_{j}\left(y_{1}, y_{2}\right) x_{1}^{j}
\end{aligned}
$$


where each $\bar{F}_{j}\left(y_{1}, y_{2}\right)$ is a homogeneous polynomial of weight degree $M:=\frac{k}{2}(n-j)$. Indeed, the degree of $\bar{F}_{j}\left(y_{1}, y_{2}\right)$ is

$$
l_{1}+l_{2}+l l_{4}+(k-l) l_{4}=l_{1}+l_{2}+k l_{4} .
$$

using that $l_{1}+l_{2}=n-\frac{k}{2}\left(l_{3}+l_{4}\right)$ and $l_{3}-l_{4}=j$ we can rewrite the above expression as

$\frac{k}{2} n-\frac{k}{2}\left(l_{3}+l_{4}\right)+k l_{4}=\frac{k}{2} n-\frac{k}{2} l_{3}+\frac{k}{2} l_{4}=\frac{k}{2} n+\frac{k}{2}\left(l_{4}-l_{3}\right)=\frac{k}{2}(n-j)$.

Note that system (8) is completely integrable with the first integrals

$$
H_{1}=\frac{y_{2}^{k-l}}{x_{1}} \quad \text { and } \quad H_{2}=\frac{y_{1}^{l+1}}{l+1}+\frac{(-1)^{l+k / 2} x_{1}^{2}}{2 \alpha(k-l+1) y_{2}^{k-l-1}} .
$$

Hence

$$
\begin{aligned}
\bar{F} & =\sum_{j_{1}, j_{2}} F_{j_{1}, j_{2}}\left(\frac{y_{1}^{l+1}}{l+1}+\frac{(-1)^{l+k / 2} x_{1}^{2}}{2 \alpha(k-l+1) y_{2}^{k-l-1}}\right)^{j_{2}} \\
& =\sum_{j_{1}, j_{2}} \sum_{m=0}^{j_{2}} \tilde{F}_{j_{1}, j_{2}, m} \frac{y_{2}^{j_{1}(k-l)-m(k-l-1)} y_{1}^{(l+1)\left(j_{2}-m\right)}}{x_{1}^{j_{1}-2 m}} .
\end{aligned}
$$

where

$$
\tilde{F}_{j_{1}, j_{2}, m}=F_{j_{1}, j_{2}}\left(\begin{array}{c}
j_{2} \\
m
\end{array}\right) \frac{(-1)^{m(l+k / 2)}}{(l+1)^{j_{2}-m}(2 \alpha(k-l+1))^{m}} .
$$

Using that $\bar{F}$ must satisfy (9), we must have that for any $m=0, \ldots, j_{2}$,

$$
\left(j_{2}-m\right)(l+1)-m(k-l-1)+j_{1}(k-l)=\frac{k}{2} n+\frac{k}{2}\left(j_{1}-2 m\right)
$$

that is

which yields

$$
j_{2}(l+1)+j_{1}(k-l)=\frac{k}{2} n+\frac{k}{2} j_{1}
$$

$$
j_{2}=\frac{1}{l+1}\left(\frac{k}{2}\left(n-j_{1}\right)+l j_{1}\right) .
$$

Note that $\bar{F}$ must be a polynomial in the variables $y_{1}$ and $y_{2}$. Thus

$$
j_{1}(k-l)-\frac{k-l-1}{l+1}\left(\frac{k}{2}\left(n-j_{1}\right)+l j_{1}\right) \geq 0 .
$$

This implies that

$$
j_{1}(k-l+1) \geq(k-l-1) n \quad \text { that is } \quad j_{1} \geq \frac{k-l-1}{k-l+1} n .
$$

Moreover using again (9) we have that $j_{1} \leq n$. Therefore,

$$
\bar{F}=\sum_{(k-l-1) n /(k-l+1) \leq j_{1} \leq n} \beta_{j_{1}, n, k, l} H_{1}^{j_{1}} H_{2}^{\left(k\left(n-j_{1}\right)+2 l j_{1}\right) /(2(l+1))},
$$

with $\beta_{j_{1}, n, k, l} \in \mathbb{C}$.

To conclude the proof of Theorem 1 it is sufficient to show that $\bar{F}=0$. Indeed if $\bar{F}=0$ then any weight homogenous polynomial 
first integral with weight exponent $(1,1, k / 2, k / 2)$ and weight degree $d=k n / 2$ restricted to $H=0$ is zero and thus system (5) cannot have a weight homogenous polynomial first integral $F$ with weight exponent $(1,1, k / 2, k / 2)$ and weight degree $d=k n / 2$ independent with $H$ since otherwise when restricted to $H=0$ this first integral would not be zero.

To show that $\bar{F}=0$, we write

$$
F=F\left(y_{1}, y_{2}, x_{1}, x_{2}\right)=\bar{F}+\left(x_{2}+\frac{2 \alpha(-1)^{l+k / 2} y_{1}^{l} y_{2}^{k-l}}{x_{1}}\right) G,
$$

where $G$ is a weight-homogenous polynomial with weight exponent $(1,1, k / 2, k / 2)$ and weight degree $d=k n / 2$. Imposing that $F$ is a first integral of system (5), after simplifying by $x_{2}+\frac{2 \alpha(-1)^{l+k / 2} y_{1}^{l} y_{2}^{k-l}}{x_{1}}$ and then setting $x_{2}=-\frac{2 \alpha(-1)^{l+k / 2} y_{1}^{l} y_{2}^{k-l}}{x_{1}}$ we get

$$
\begin{aligned}
& \sum_{(k-l-1) n /(k-l+1) \leq j_{1} \leq n} \frac{\beta_{j_{1}, n, k, l} j_{1}(k-l)}{y_{2}} H_{1}^{j_{1}} H_{2}^{\left(k\left(n-j_{1}\right)+2 l j_{1}\right) /(2(l+1))} \\
& +\sum_{(k-l-1) n /(k-l+1) \leq j_{1} \leq n} \frac{\beta_{j_{1}, n, k, l}\left(k\left(n-j_{1}\right)+2 l j_{1}\right)(-1)^{l+k / 2}(1-k+l) x_{1}^{2} y_{2}^{l-k}}{4 \alpha(l+1)(1+k-l)} \\
& \cdot H_{1}^{j_{1}} H_{2}^{\left(k\left(n-j_{1}\right)+2 l j_{1}\right) /(2(l+1))-1}+\frac{2(k-l)(-1)^{l+k / 2} y_{1}^{l} y_{2}^{k-l} \alpha}{x_{1} y_{2}} G \\
& +x_{1} \frac{\partial G}{\partial y_{1}}-\frac{2 \alpha(-1)^{l+k / 2} y_{1}^{l} y_{2}^{k-l}}{x_{1}} \frac{\partial G}{\partial y_{2}}-2 \alpha(-1)^{l+k / 2}(k-l) y_{1}^{l} y_{2}^{k-l-1} \frac{\partial G}{\partial x_{1}} \\
& =0 .
\end{aligned}
$$

Solving this partial differential equation we obtain

$$
\begin{aligned}
G= & \frac{(-1)^{l+k / 2+1}(k-l)}{2 \alpha y_{1}^{l} y_{2}} \sum_{(k-l-1) n /(k-l+1) \leq j_{1} \leq n} j_{1} \beta_{j_{1}, n, k, l} H_{1}^{j_{1}} H_{2}^{\left(k\left(n-j_{1}\right)+2 l j_{1}\right) /(2(l+1))} \\
& +\frac{(-1)^{l+k / 2}(1-k+l)^{2} x_{1}^{5}}{8(1+k-l) \alpha^{2} y_{1}^{l}\left(2 \alpha y_{1}^{l+1}(k-l+1) y_{2}^{k-l-1}+(-1)^{l+k / 2}(l+1) x_{1}^{2}\right)} \\
& \sum_{j_{j_{1}, n, k, l}\left(k\left(n-j_{1}\right)+2 l j_{1}\right) H_{1}^{j_{1}} H_{2}^{\left(k\left(n-j_{1}\right)+2 l j_{1}\right) /(2(l+1))}} \sum^{(k-l-1) n /(k-l+1) \leq j_{1} \leq n} \\
& +x_{1} K\left(H_{1}, H_{2}\right),
\end{aligned}
$$

where $K$ is any function in the variables $H_{1}$ and $H_{2}$. Since $G$ must be a polynomial in the variables $y_{1}$ and $y_{2}$ and $k\left(n-j_{1}\right)+2 l j_{1} \neq 0$ due to the fact that $j_{1} \leq n$, we must have $\beta_{j_{1}, n, k, l}=0$, i.e. $\bar{F}=0$. This concludes the proof of the theorem. 


\section{ACKNOWLEDGEMENTS}

The first author is partially supported by a MINECO/FEDER grant MTM2008-03437 and MTM2013-40998-P, an AGAUR grant number 2014SGR-568, an ICREA Academia, the grants FP7-PEOPLE-2012IRSES 318999 and 316338, grant UNAB13-4E-1604.

The second author is supported by Portuguese national funds through FCT - Fundação para a Ciência e a Tecnologia: project PEst-OE/EEI/ LA0009/2013 (CAMGSD).

\section{REFERENCES}

[1] J. Hietarinta, Direct methods for the search of the second invariant, Phys. Rep. 147 (1987), 87-154.

[2] A.J. Maciejewski and M. Przybylska, Darboux points and integrability of Hamiltonian systems with homogeneous polynomial potential, J. Math. Phys. 46 (2005), no. 6, 062901.

[3] K. Meyer, Generic Hamiltonian dynamical systems are neither integrable nor ergodic, Memoirs of the American Mathematical Society. 144, American Mathematical Society, Providence, R.I., 1974.

1 Departament de Matemàtiques, Universitat Autònoma de Barcelona, 08193 Bellaterra, Barcelona, Catalonia, Spain

E-mail address: jllibre@mat.uab.cat

2 Departamento de Matemática, Instituto Superior Técnico, Universidade Técnica de Lisboa, Av. Rovisco Pais 1049-001, Lisboa, PorTUGAL

E-mail address: cvalls@math.ist.utl.pt 